\title{
La végétation des sols polygonaux aux étages alpin supérieur et subnival en Valais (Alpes centro-occidentales, Suisse)
}

\author{
Claude Béguin', Mélanie Progin Sonney et Michel Vonlanthen \\ Université de Fribourg, Département de Géosciences, Chemin du Musée 4, \\ CH-1700 Fribourg \\ 1 Adresse présente: CH-2067 Chaumont; e-mail: clfbeguin@bluewin.ch
}

Manuscrit accepté le 6 mars 2006

\begin{abstract}
Béguin Cl., Progin Sonney M. and Vonlanthen M. 2006. The vegetation of polygonal soils at the upper alpine and subnival belts in Switzerland. Bot. Helv. 116: 41-54.

Polygonal soils are typical formations of arctic regions but occur locally at high elevation in the Alps. A vegetation survey of sites with polygonal patterning of soils in the Western Swiss Alps revealed a clear distinction between (1) periglacial polygonal soils, whose structure is created by frost action, and whose occurrence is limited to the subniveal belt (2700-3000 m a.s.l.) and (2) soils whose polygonal structures are mere contraction cracks created by desiccation, which are found in the alpine belt (2300-2750 m a.s.l). Two new plant associations characteristic of these sites are described: periglacial polygonal soils of the subniveal belt are colonized by the association Saxifrago oppositifoliae-Poetum alpinae ass. nov. of the alliance Drabion hoppeanae. Conversely, soils with polygonal contraction cracks shelter the association Epilobio anagallidifoliiSaginetum saginoidis ass. nov., which is related to the alliance Salicion herbaceae. We describe the particular geomorphological and climatic factors leading to the local formation of these soil types with their specialised vegetation. We conclude that their future existence is strongly threatened by climate warming.
\end{abstract}

Key words: Alpine synecology, cryoturbation, Drabion hoppeanae, geomorphology, permafrost, Salicion herbaceae.

\section{Introduction}

Par leur physionomie, les sols polygonaux ont toujours attiré l'attention des observateurs, en particulier dans les zones arctiques où ils sont assez fréquents (Wasburn 1979; Pissart 1987; French 1996). Quelques auteurs se sont penchés sur leur végétation (Jonasson et Sköld 1983; Gold et Bliss 1995; Cannone et al. 2004). En dehors des zones arctiques, et en particulier dans les Alpes, ces formes géomorphologiques ont une 
extension très restreinte. Elles n'ont que rarement été étudiées (Pissart 1973). En Suisse, seul Campiche (1986) s'y est intéressé dans le cadre d'une étude de phénomènes périglaciaires en marge de la Plaine Morte au-dessus de Montana en Valais.

L'expression «sols polygonaux» est utilisée en géomorphologie pour désigner des objets d'aspect semblable mais obéissant à des processus de formation différents $(\mathrm{R}$. Delaloye, E. Gerber, W. Haeberli, M. Monbaron, A. Pancza, J.-P. Portmann, E Reynard, communication orale). Aux étages alpin et subnival dans les Alpes, on peut distinguer deux types de sols polygonaux entre lesquels existent des convergences de formes. D'une part, les sols polygonaux structurés où s'effectue un triage du matériel grossier par le gel, et d'autre part, les sols polygonaux avec fentes de dessiccation, sans tri par le gel. Les sols de la première catégorie seront désignés ici comme des sols polygonaux alpins au sens strict.

A notre connaissance, aucune étude phytosociologique n'a été réalisée en Suisse pour décrire cette curiosité naturelle. Dans ce travail, nous présentons une analyse «fine» de la végétation en cherchant à comprendre le déterminisme même de cette végétation. Si l'écologie des espèces rencontrées est connue d'un point de vue autécologique, l'approche synécologique est méconnue. A ce niveau d'intégration, nous cherchons à compléter et préciser les facteurs édaphiques déterminants et plus spécialement les dispositifs géomorphologiques qui entourent les deux types de sols polygonaux.

\section{Méthodes}

Sites étudiés

Le choix des sites étudiés a été dicté par la rareté des stations connues de sols polygonaux dans les Alpes (Fig. 1). C'est surtout dans le Haut-Val de Réchy et la région de Lona dans les Alpes pennines (Valais, Suisse) que l'on trouve des sols polygonaux bien développés. Nous y avons effectué une vingtaine de relevés en nous basant sur les cartes géomorphologiques au 1:5000 de Tenthorey (1993) et de Gerber (1994). Quelques relevés supplémentaires dans des situations semblables proviennent de la rive droite du Rhône (Plaine Morte et Torrenthorn, Alpes bernoises) ainsi que du Grand Galibier (Hautes Alpes, France). En outre, des observations complémentaires ont été réalisées dans les régions du Gornergrat (Valais), des Grandes Gouilles au pied du Wildhorn (Valais) et au Col du Palet au dessus de Tignes (France). L'altitude des sites varie de 2380 à $2995 \mathrm{~m}$.

Le Val de Réchy et la région de Lona font partie des nappes pennines moyennes et supérieures du Valais central. Les sols polygonaux rencontrés reposent sur un substratum géologique formé essentiellement de schistes et de quartzites sur lesquels s'accumulent divers matériaux d'alluvionnement provenant de l'altération des versants susjacents et de dépôts éoliens. Le climat est de type subcontinental d'altitude (environ $1300 \mathrm{~mm}$ de précipitations neigeuses et pluvieuses). La force et la fréquence des vents ainsi que les alternances de gel et de dégel sont importantes. Les stations de relevés complémentaires (cf. Annexe) présentent des conditions édaphiques et climatiques proches de celles du Val de Réchy et de la région de Lona.

Analyse de la végétation

La végétation a été étudiée au moyen de relevés phytosociologiques (Braun-Blanquet 1964; Guinochet 1973) sur des surfaces homogènes de 1.5 à 18 m2. La nomencla- 


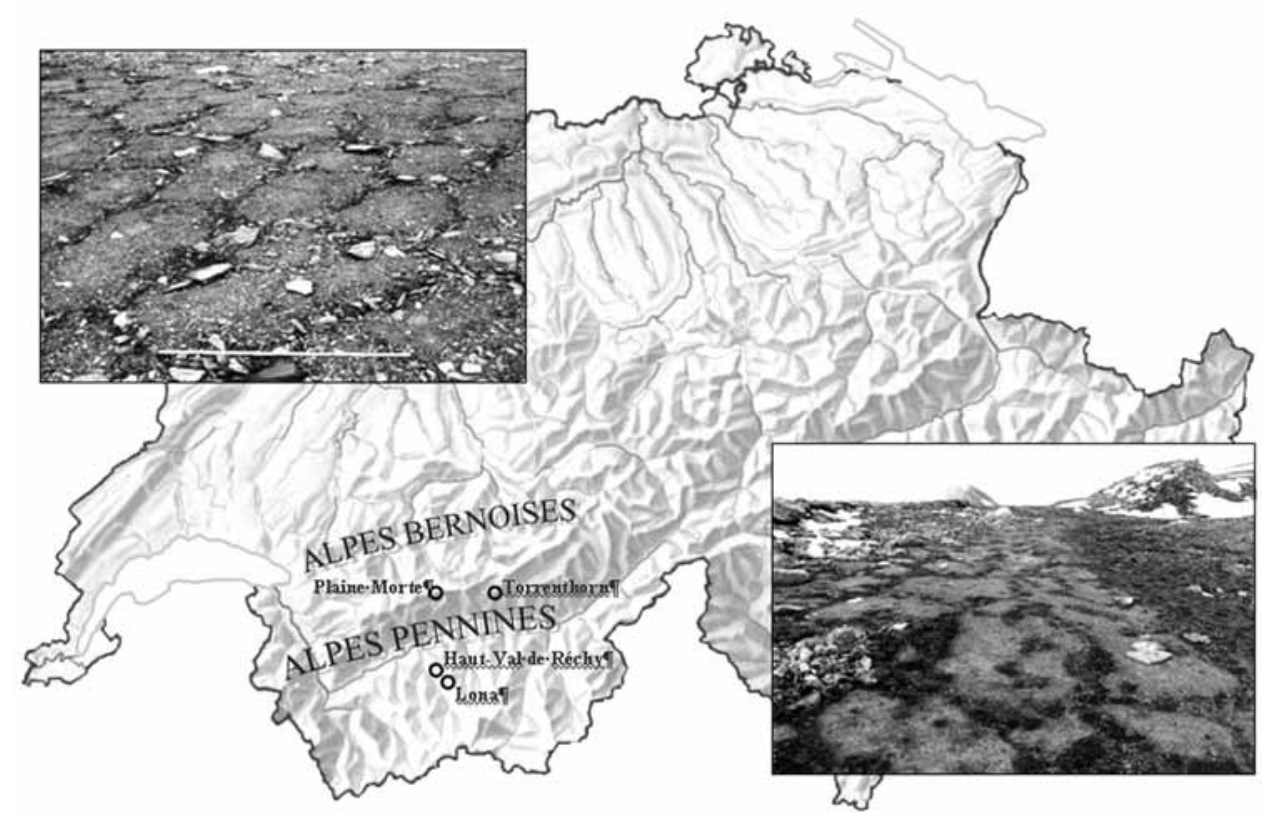

Fig. 1. Localisation des sols polygonaux s.l. étudiés dans les Alpes pennines et les Alpes bernoises. En encarté: physionomie et diversité de deux stations dans la région du Haut Val de Réchy (en haut à gauche : relevé 3, tableau I ; en bas à droite : relevé 8, tableau I).

ture des plantes vasculaires suit Lauber et Wagner (2001). La position synsystématique des relevés a été évaluée sur la base de Theurillat et al. (1995) en appliquant le critère suivant: «En principe, une association végétale doit posséder au moins une espèce caractéristique. Néanmoins, on est parfois en présence de groupements très bien individualisés, stables et homogènes qui, floristiquement et écologiquement, ne peuvent se rattacher à aucune association connue. Dans ces cas extrêmes, s'il apparaît qu'un tel groupement mérite effectivement le statut d'association, on le caractérise par une combinaison caractéristique d'espèces» (Theurillat et Matthey 1987).

Les exigences écologiques de différents groupes d'espèces (combinaisons caractéristiques d'associations, espèces différentielles de sous-associations) ont été décrites à l'aide des valeurs bioindicatrices de Landolt (1977). Les indices moyens de ces groupes d'espèces (présentes en 2004) permettent de rechercher une différentiation écologique entre les sites des relevés. Ils servent également de base pour individualiser les associations et sous-associations décrites dans ce travail par rapport à d'autres unités de végétation.

Pour les 11 stations du tableau 3, nous avons reporté, dans un rayon de 20 m entourant les relevés, les formes et les processus géomorphologiques selon Gerber (1994). L'analyse du tableau a révélé deux groupements végétaux distincts, correspondant aux deux types de sols polygonaux (Tab. 1, sols polygonaux au sens strict; Tab. 2, sols avec fentes de dessiccation). Quelques relevés intermédiaires (groupe B dans Tab. 3) n’ont pas été inclus dans les tableaux de végétation. Les observations géomorphologiques 
ont été mises en rapport avec la végétation en subdivisant les 11 relevés selon les associations végétales. Le tableau 3 montre une étroite corrélation entre la phytosociologie et la géomorphologie.

\section{Résultats et discussion}

Saxifrago oppositifoliae-Poetum alpinae ass. nov. sur sol polygonal s. str.

\section{1) Sociologie}

La végétation des sols polygonaux cryoturbés très actifs, avec tri par le gel, de l'étage subnival se caractérise par une combinaison originale d'espèces de chamaéphytes et d'hémicryptophytes dont plus de la moitié sont d'origine arctico-alpine, les autres éléments phytogéographiques étant à rechercher essentiellement dans les Alpes et dans différentes parties d'Europe (centro- et sud-européen, Tab. 1). Le recouvrement total de la végétation est faible (0 à $30 \%$ ). Dans quelques cas, les sols polygonaux semblent offrir des conditions de vie trop rudes et n'abritent alors aucune plante vasculaire.

Les sols polygonaux s. str. présentent deux types de micro-sites : la partie centrale des polygones est constituée de matériaux fins, alors que leur bordure est caillouteuse, avec des plaquettes redressées par le gel. Un essai de relevés séparés dans ces deux parties n'a cependant pas révélé de discrimination végétale suffisante pour les traiter comme des «hypogéosigmassociations» (petites unités paysagères, Theurillat 1992). Les sols polygonaux décimétriques doivent donc être considérés comme des surfaces floristiquement homogènes malgré leur hétérogénéité microgéomorphologique. La combinaison caractéristique des espèces et des conditions de vie extrêmes (Körner 2003 ) permettent d'individualiser une nouvelle association, le Saxifrago oppositifoliaePoetum alpinae ass. nov. Hoc loco (holotypus relevé 3, Tab. 1).

La combinaison caractéristique d'espèces de l'association est la suivante : Poa alpina, Saxifraga oppositifolia, Sagina saginoides et Pritzelago alpina s.l. (Tab. 1). Ces espèces peuvent croître aussi bien dans la bordure caillouteuse des polygones que dans la partie centrale formée essentiellement de particules fines. Elles présentent une certaine amplitude écologique vis à vis du calcaire. Leur valeur écologique moyenne d'humidité $(\mathrm{H})$ atteint 3.6 sur l'échelle de Landolt, traduisant le caractère humide du milieu (Tab. 1).

Poa alpina, souvent sous sa forme vivipare, marque la physionomie des stades pionniers de l'association (recouvrement total de 0 à 20\%). Il n'apparaît généralement pas à vitalité réduite ou avec un retard phénologique (Tab. 1). Saxifraga oppositifolia et Pritzelago alpina s.l. sont des espèces pionnières de lumière, de terrains caillouteux riches en bases et exposés au gel (Oberdorfer 1994). Elles se rencontrent avant tout dans les associations d'éboulis. Pritzelago alpina s.l. traduit le caractère humide du milieu puisque cette espèce peut encore être présente dans des groupements fontinaux. Il en va de même pour Carex parviflora, Ranunculus glacialis ou Saxifraga stellaris. Sagina saginoides est une espèce indicatrice d'humidité variable qui croît sur des sols plutôt secs, mais détrempés lors de pluies prolongées ou au moment de la fonte des neiges (Grabherr et Mucina 1993). Elle est absente des éboulis en mouvement et occupe préférentiellement la partie centrale des polygones.

Sous-associations

On peut distinguer provisoirement deux sous-associations dans le Saxifrago-Poetum alpinae en se basant sur les plantes vasculaires. Une sous-association à Cerastium 
latifolium et une sous-association à Silene exscapa. Les espèces différentielles des sousassociations se développent essentiellement à l'intérieur des bordures polygonales (sans matrice).

Saxifrago-Poetum cerastietosum latifolii (holotypus relevé 3, Tab.1). Les espèces différentielles sont des plantes d'éboulis calcaires, donc de milieux ouverts, sans compétition pour la lumière. Les sols contiennent peu de matière organique. Leur végétation pionnière (degré de recouvrement: 0 à $20 \%$ ) est pauvre en espèces (2-10). La sousassociation cerastietosum se situe souvent sur de grands lobes de solifluxion actifs ou sur de larges terrasses de sols flués. Parfois, les roches schistées entrent en contact avec des roches compactes comme le Kimméridgien (relevés 4, 5 ; Tab. 1).

Saxifrago-Poetum silenetosum exscapae (holotypus relevé 8, Tab. 1). Une bonne partie des espèces de la sous-association silenetosum sont des plantes d'éboulis, alors que Silene exscapa, Leucanthemopsis alpina et Ligusticum mutellinoides ont leur optimum dans les pelouses acides du Caricetalia curvulae, c'est à dire sur des sols moins instables, plus mûrs et plus chauds. Le degré de recouvrement de la végétation dépasse celui de la sous-association précédente (25 à 30\%), et le nombre d'espèces est plus élevé (13-15). Cette sous-association se rapproche de l'Artemisio-Saxifragetum (Richard et al. 1993) avec la présence de Saxifraga muscoides et l'important recouvrement de Saxifraga oppositifolia (arêtes de l'étage subnival sur calcschistes, exposés et peu enneigés). Les valeurs bioindicatrices des espèces (Tab. 1) montrent que la sous-association à silène se différencie de la sous-association à céraiste par des valeurs plus élevées de continentalité et d'acidité et des valeurs moins élevées d'humidité et de porosité.

Position synsystématique

La végétation des sols polygonaux s.str. appartient à la classe des Thlaspietea rotundifolii, végétation des éboulis s.l. Avec la présence de Draba hoppeana, Saxifraga biflora, S. muscoides, Herniaria alpina et Minuartia sedoides, ce type de végétation peut se rattacher à l'ordre des Drabetalia hoppeanae et à l'alliance du Drabion hoppeanae qui correspond à des éboulis de calcschistes s.l. L'association présente des analogies avec le groupement à Saxifraga oppositifolia, Saxifraga androsacea et Arabis coerulea en bas de pente et à très long enneigement (groupement local formé de 3 relevés du Val de Réchy; Richard et al. 1993). Toutefois, le Saxifrago-Poetum ne correspond pas à des éboulis proprement dit mais à des alluvions cryoturbées où les espèces d'éboulis rencontrent une niche écologique refuge sous la forme de fragments rocheux triés et soulevés par le gel.

\section{2) Synécologie}

Les sols polygonaux s.str. du Saxifrago-Poetum correspondent à des situations ponctuelles de type replats, épaulements, selles, cols de tête ou cols de flanc (Fig. 2a). Il s'agit d'endroits précis du modelé périglaciaire où se forment des sols gélifs qui contiennent beaucoup d'eau et où les pores sont fins. Ce sont des stations venteuses où un transect convexe coupe un transect concave (Fig. 2a). Un tel dispositif favorise d'un côté la formation de névés sus-jacents et d'un autre côté le déneigement rapide du sol par des vents secs, forts et fréquents. Il accentue ainsi l'effet mécanique du gel. Par les variations de volume qu'il impose au sol, le gel provoque des mouvements de particules, une fragmentation du sol et le déchaussement, voire la rupture, du système radiculaire des plantes (Duchaufour 1970). L'«effet de col» avec ses brusques chutes de température favorise la formation d'aiguilles de glace (Brink et al. 1967); ceci déplace et trie les plaquettes éparses dans les chenaux (Brink et al. 1967; Pissart 1987), condui- 

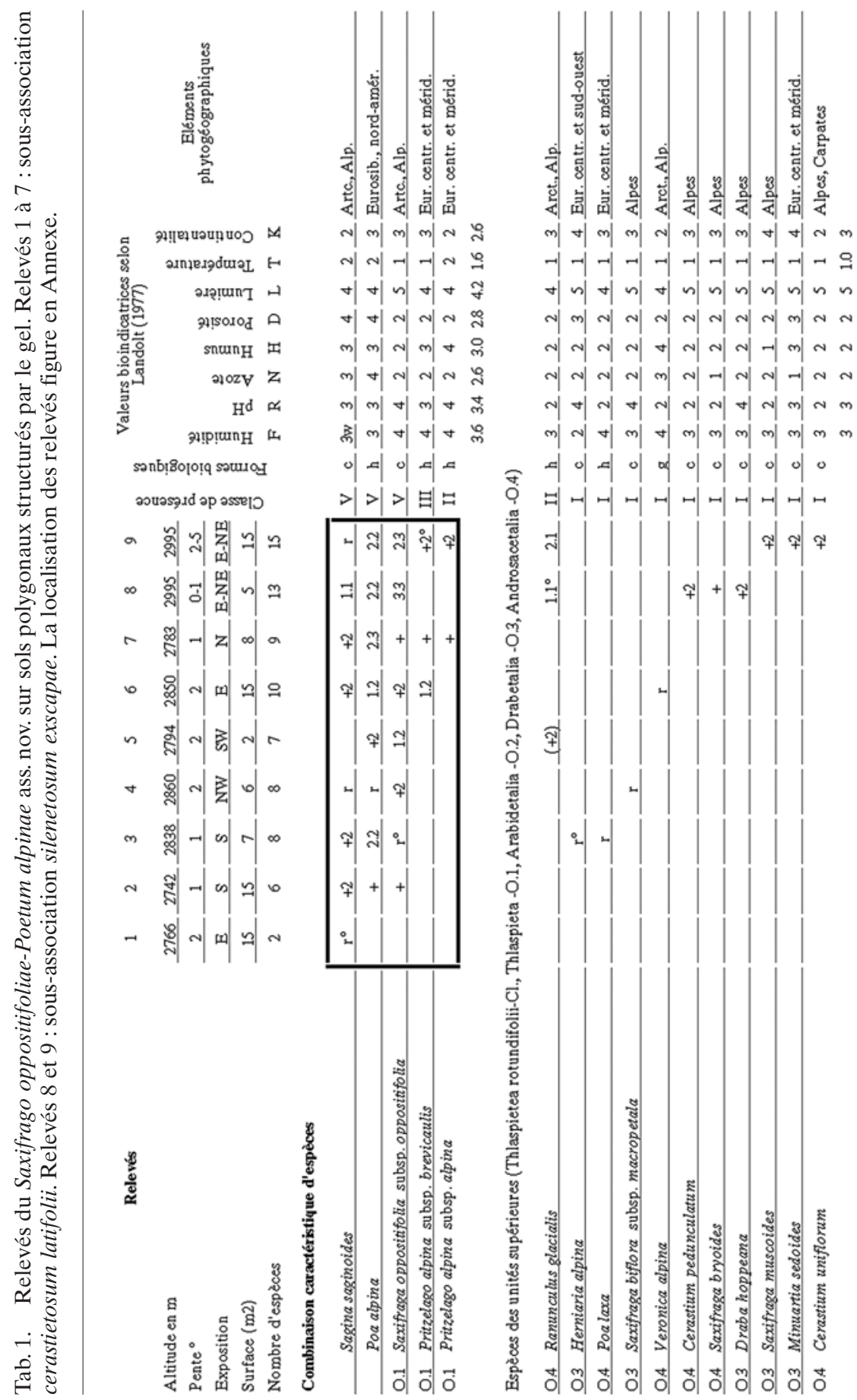


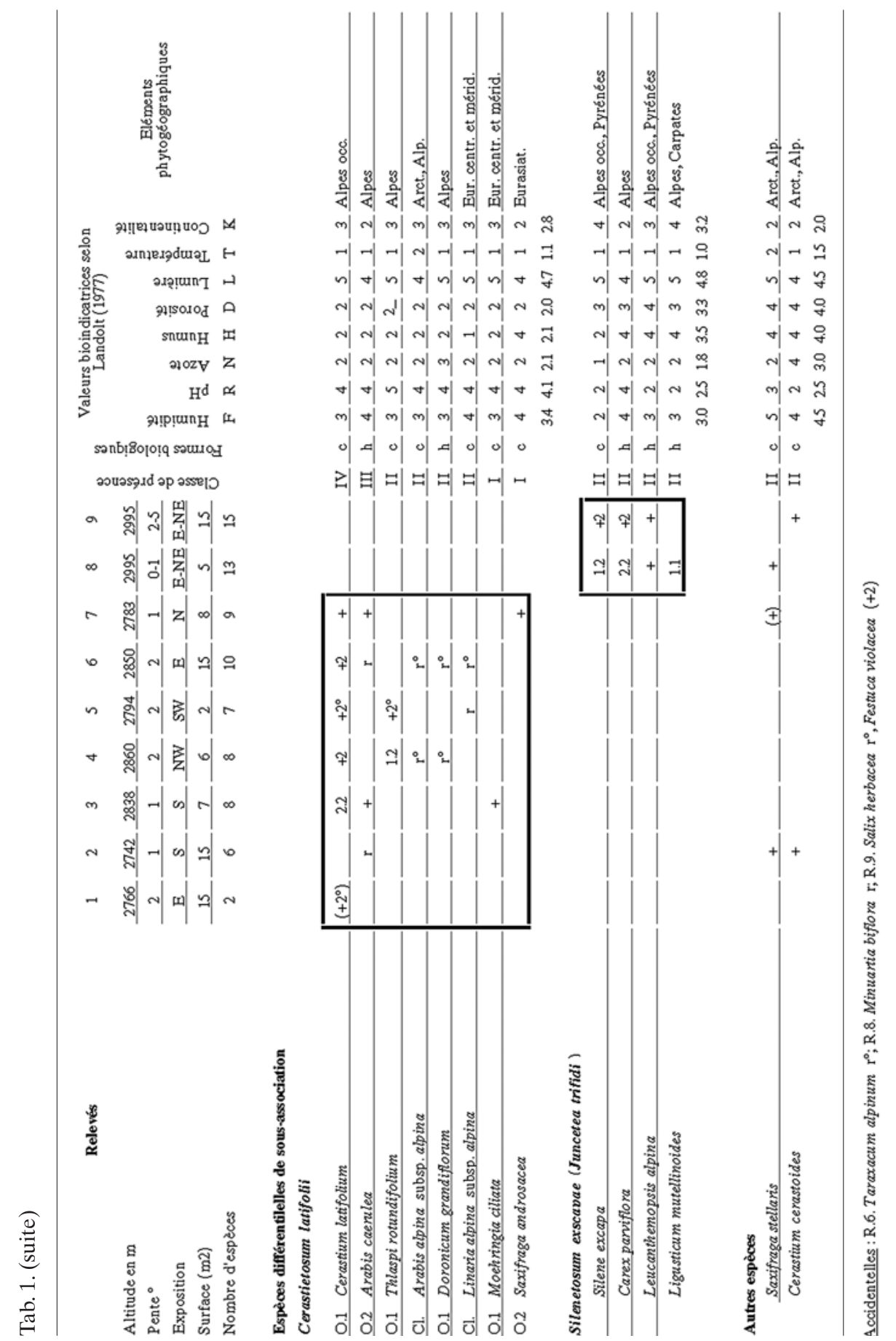


(a)

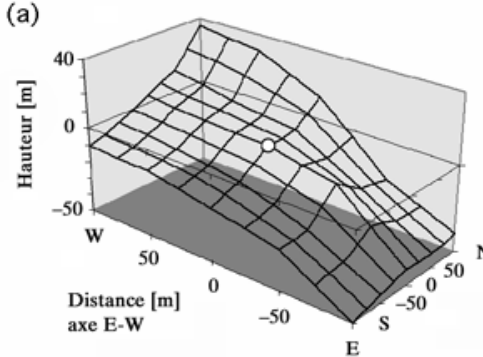

(b)

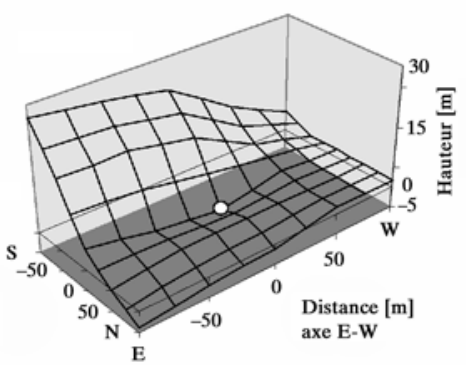

Fig. 2. Dispositif géomorphologique caractéristique (a) du Saxifrago-Poetum comportant un transect convexe et un transect concave (relevé 8 du tableau 1) et (b) de l'Epilobio-Saginetum comportant un double transect concave (relevé 4 du tableau 2). Les distances horizontales (deux transects perpendiculaires) et verticales sont mesurées à partir de la station du relevé.

sant à un arrangement géométrique des pierres. D'une manière générale, peu d'espèces peuvent supporter un stress mécanique aussi intense.

D'après nos observations, la combinaison de trois processus écologiques principaux déterminent l'emplacement et la formation des sols polygonaux alpins s. str.

(a) L'alimentation en eau par un dispositif d'horizons humides avec ruissellement diffus, voire concentré, en surface ou en profondeur. L'eau provient d'un névé sus-jacent fondant lentement jusqu'à la fin de la période de végétation. Grâce à ce début d'alluvionnement se forme un sol minéral subhorizontal gorgé d'eau sur lobes de solifluxion presque stabilisés. La proximité d'un petit lac à niveau fluctuant durant l'été peut jouer un rôle favorable, complémentaire ou compensatoire en remplaçant l'eau de fonte d'un névé asséché (station du relevé 1 par exemple).

(b) La gélifraction sur des affleurements rocheux sus-jacents schistés, microfissurés, gélifs et très actifs. Ces bancs débitent une régolithe en plaquettes de toutes tailles et une fraction minérale fine pouvant se mélanger à des dépôts éoliens. Le sol est donc formé en grande partie de fragments de la roche mère. Il s'agit d'une simple désagrégation physique en climat très froid.

(c) La géliturbation intense et fréquente est accentuée par compression et déflation du vent (congélation, évaporation). La présence de vent sec favorise l'évaporation de l'eau des sols à texture limoneuse, d'où une perte énergétique supplémentaire importante. Il en résulte un gonflement du sol et la formation lente de lentilles de glace avec soulèvement et déplacement des fragments de schistes (Smith 1987). Finalement, les fragments s'accumulent dans les chenaux, fissures de retrait ou gélivures formant des polygones décimétriques, avec plus ou moins de cailloux redressés verticalement.

Epilobio anagallidifolii-Saginetum saginoidis ass. nov.

1) Sociologie

A l'étage alpin, les rives de lacs peu profonds, périodiquement asséchés par évaporation et percolation, présentent parfois des fentes de dessiccation polygonales. Ces 
'pseudosols polygonaux' sans tri par le gel sont le site d'une association végétale spécialisée différente, l'Epilobio anagallidifolii-Saginetum saginoidis ass. nov. hoc loco (holotypus relevé 1, Tab. 2). Le tableau 2 met en évidence un fort degré de parenté entre les relevés, qui comprennent en moyenne près de 12 espèces.

La combinaison caractéristique d'espèces est la suivante : Sagina saginoides, Cerastium cerastioides, Gnaphalium supinum et Ranunculus confervoides. Toutes ces espèces sont des arctico-alpines. $R$. confervoides est relativement rare et fortement liée aux eaux peu profondes. Le plus souvent submergée, elle traduit bien le caractère lacustre de cette association.

\section{Position synsystématique}

L'association des pseudosols polygonaux présente une certaine convergence floristique avec le Saxifrago-Poetum alpinae, par la présence notamment de Sagina saginoides, Poa alpina, Saxifraga oppositifolia, S. stellaris, Cerastium cerastioides, Carex parviflora. Toutefois, les espèces des éboulis calcaires présentes dans les sols polygonaux s.str. sont faiblement représentées dans l'Epilobio-Saginetum, si l'on excepte Saxifraga oppositifolia et la présence accidentelle de Pritzelago alpina. Par ailleurs, les relevés des pseudosols polygonaux se différencient par la constance de Poa supina, Cardamine alpina et Veronica alpina.

L'Epilobio-Saginetum se range aisément dans la classe des Salicetea herbaceae. Par contre, le choix de l'alliance (Salicion herbaceae, Salici herbaceae-Caricion lachenalii) est moins évident. En effet, les principales espèces caractéristiques et dominantes du Salicion herbaceae sont absentes de l'Epilobio-Saginetum: Salix herbacea, Alchemilla pentaphyllea, Carex foetida, Arenaria biflora, Sibbaldia procumbens (Grabherr et Mucina 1993; Theurillat et al. 1995). De même, les espèces caractérisant le Salici-Caricion lachenalii manquent: Carex lachenalii, Salix foetida, S. hastata, S. helvetica (Béguin et Theurillat 1983). Toutefois, l'association est proche du Poo-Cerastietum Sörinki ex Oberdorfer 1962 (synonyme : Poetum alpinae Braun 1913 non Rübel; Grabherr et Mucina 1993), groupement nitrophile des étages subalpins supérieur et alpin inférieur, mais habituellement rangé dans le Salicion herbaceae. De ce fait, il est préférable de considérer l'Epilobio-Saginetum également comme un groupement initial permanent du Salicion herbaceae, à caractère nitrophile (Cerastium cerastioides, Poa alpina, $P$. supina), manifestant ainsi une forte tendance vers le Poion alpinae et vers les éboulis fins et humides de l'Androsacion (Epilobium anagallidifolium, Saxifraga oppositifolia et S. stellaris) plutôt que comme un groupement oligotrophe du Salici-Caricion lachenalii, alliance floristiquement et écologiquement proche des groupements fontinaux et des bas-marais oligotrophes.

\section{2) Synécologie}

L'Epilobio-Saginetum est une association spécialisée qui repose sur des dépôts vaseux suintants en bordure des petits lacs alpins entre les limites moyennes des hautes eaux et des basses eaux (submersion temporaire). Le dispositif géomorphologique (Fig. 2b) fait ressortir une position concave, contrairement au dispositif du SaxifragoPoetum qui présente un certain degré de convexité à proximité des lignes de crête. Ce type de végétation apparaît comme le vicariant à l'étage alpin (2400-2800 m) du Veronico scutellatae-Alopecuretum aequalis (Béguin et Theurillat 1981a) du bord des petits lacs de l'étage subalpin supérieur. La moitié des espéces de l'Epilobio-Saginetum sont des espèces d'origine arctico-alpine qui affectionnent les terrains neufs, rajeunis, propices au développement optimal d'espèces peu compétitives. 


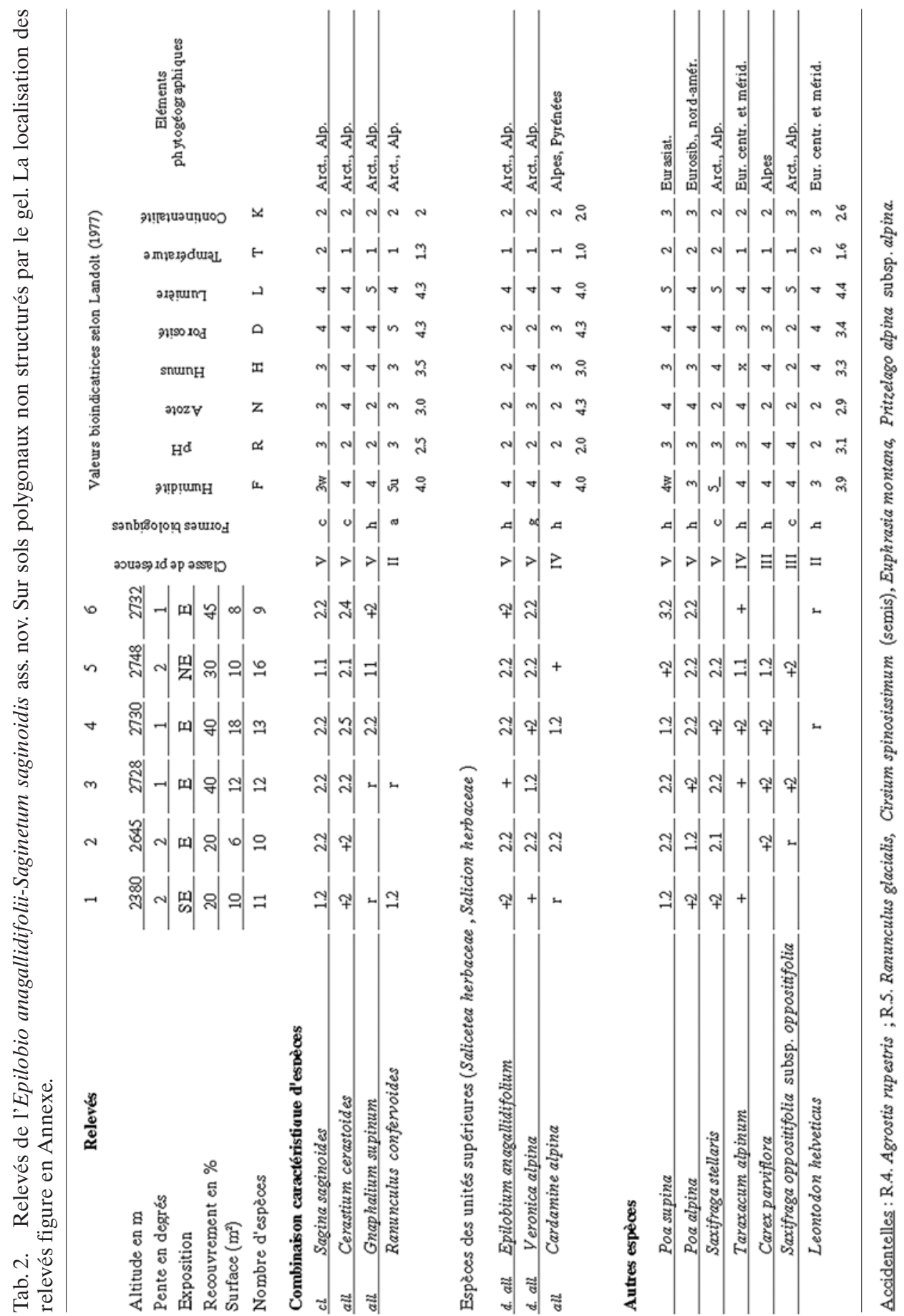


Nos observations montrent que la combinaison de trois facteurs écologiques principaux explique le déterminisme de l'association :

(a) Le massage aquatique dû au flux et au reflux de l'eau ainsi qu'à l'intensité des vagues. Ce processus est particulièrement actif au moment de la fonte de la glace. Il détermine ainsi une zone de battement et creuse des niches d'érosion. La force et la fréquence des vents de la zone alpine jouent un rôle important. Les courants longitudinaux de déferlement provoquent des mouvements de la fraction minérale fine en bordure de la beine lacustre (Raemy et Huber 1990) et le transport d'argiles par les courants de retour au large rajeunissent constamment la rive peu profonde sur laquelle se développe l'association. Ce processus d'érosion régressive bloque la dynamique d'un développement de la végétation vers les groupements de combes à neige évolués et stables. En particulier le déferlement des vagues sur la berge constitue un obstacle physique inhibant l'expansion des saules (chamaéphytes et nanophanérophytes), ainsi que plusieurs autres espèces du Salicion herbaceae.

(b) Un sol argileux à structure dispersée. Lorsque les sols à structure dispersée, riches en argile sont soumis à un fort retrait, il se produit une fissuration de la masse qui se compartimente en éléments anguleux (structure par fragmentation), ce qui augmente temporairement l'aération et la perméabilité (Duchaufour 1970). Les périodes de sécheresse provoquent des fentes de retrait (argiles à mauvaise structure) qui s'estompent plus ou moins en fonction des fluctuations du niveau du lac et des horizons humides, voire du ruissellement diffus. En outre, le sol compact est non gélif, une partie de l'eau retenue entre les feuillets argileux n'étant pas mobilisable. Il y a ainsi gonflement partiel des argiles mais sans formation de lentilles de glace lors des gels nocturnes.

(c) Le pâturage. Naturellement, les milieux aquatiques de l'étage alpin sont oligomésotrophes. La présence du bétail, qui utilise ces milieux comme abreuvoirs, provoque un apport important de nutriments qui peuvent être fixés grâce aux argiles, d'où la dominance des espèces mésotrophes et eutrophes.

\section{Conclusions}

Dans les Alpes centro-occidentales, les sols polygonaux se rencontrent dans des dispositifs géomorphologiques bien déterminés (Fig. 2; Tab. 3). De telles structures sont rares et n'occupent que de petites surfaces de l'ordre du décamètre carré. Les deux nouvelles associations végétales (Saxifrago oppositifoliae-Poetum alpinae et Epilobio anagallidifolia-Saginetum saginoidis) mises en évidence permettent de différencier et de caractériser le complexe des sols polygonaux aux étages alpin et subnival.

Nos observations ont montré que l'existence des deux associations spécialisées dépend d'un régime hydrique et climatique particulier. Leur existence est donc fortement menacée par le réchauffement climatique en cours qui risque de modifier ces facteurs déterminants (Beniston 1994; Beniston et al. 1996; Haeberli and Beniston 1998). Sur la base de nos observations et des travaux de Theurillat et al. (1998), Guisan et al. (1998), Guisan et Theurillat (2000), Theurillat et Guisan (2001), nous supposons qu'un climat plus chaud et plus sec pourrait favoriser l'évolution du Saxifrago-Poetum vers les groupements de pelouse: (Seslerietalia albicantis pour la sous-association cerastieto- 
Tab. 3. Processus et formes géomorphologiques présents à proximité des sols polygonaux s.l. (dans un rayon de $20 \mathrm{~m}$ ). La localisation des relevés figure en Annexe.

\begin{tabular}{|c|c|c|c|c|c|c|c|c|c|c|c|}
\hline \multirow{2}{*}{$\begin{array}{c}\text { Sols polygonaux } \\
\text { Stations }\end{array}$} & \multicolumn{3}{|c|}{ A } & \multicolumn{3}{|c|}{ B } & \multicolumn{5}{|c|}{ C } \\
\hline & 1 & 2 & 3 & 4 & 5 & 6 & 7 & 8 & 9 & 10 & 11 \\
\hline \multicolumn{12}{|l|}{ GEOMORPHOLOGIE } \\
\hline PERIGLACIAIRE & + & + & + & + & + & + & & & & & \\
\hline Sols polygonaux (avec tri) & + & + & + & + & + & + & & & & & \\
\hline Sols striés & + & + & + & + & + & + & & & & & \\
\hline GRAVITAIRE ET PEDOGENETIQUE & & & & + & + & + & & & & & \\
\hline Sols dallés & & & & + & + & + & & & & & \\
\hline Ecoulement temporaire & & & & + & + & + & & & & & \\
\hline Lac à niveau variable & & & + & + & + & + & + & + & + & + & + \\
\hline Roche moutonnée & & & & + & + & + & + & + & + & + & + \\
\hline GRAVITAIRE ET PERIGLACIAIRE & & & & & & & + & + & + & + & + \\
\hline ANTROPOZOOGENE & & & & & & & + & + & + & + & + \\
\hline Cône et talus d'éboulis & & & & & & & + & + & + & + & + \\
\hline Sols polygonaux (sans tri) & & & & & & & + & + & + & + & + \\
\hline PEDOGENETIQUE & + & + & + & & + & + & + & + & + & + & + \\
\hline Barre rocheuse & + & + & + & + & + & + & + & + & + & + & + \\
\hline Roche en place & + & + & + & + & + & + & + & + & + & + & + \\
\hline Lobes de solifluxion & $(+)$ & & + & + & & + & + & + & + & + & + \\
\hline \multicolumn{12}{|c|}{$\begin{array}{l}\text { MAJUSCULES : processus géomorphologiques } \\
\text { Minuscules : formes géomorphologiques }\end{array}$} \\
\hline \multicolumn{12}{|c|}{$\begin{array}{l}\text { A : Sols polygonaux sensu stricto, Saxifrago-Poetum } \\
\mathrm{B} \text { : Sols polygonaux intermédiaires correspondant à un mélange de végétation } \\
\mathrm{C}: \text { Sols polygonaux de dessiccation (pseudosols polygonaux), Epilobio-Saginetum }\end{array}$} \\
\hline
\end{tabular}

sum et Caricion curvulae pour la sous-association silenetosum). En revanche, la dynamique de l'Epilobio-Saginetum devrait s'effectuer en direction du Poion alpinae ou en direction du Salicion herbaceae si les précipitations neigeuses augmentent; une augmentation des précipitations estivales rendrait moins temporaires et plus importants les petits lacs alpins favorisant ainsi un passage au Ranunculetum confervoidis (Béguin 2006).

Ces deux associations, comprenant un nombre important d'espèces arctico-alpines, risquent non seulement de disparaître de leurs emplacements actuels suite au réchauffement climatique, mais même de disparaître complètement des Alpes. En effet, d'une part la superficie potentiellement disponible pour l'étage alpin sera réduite de $66 \%$ et celle de l'étage nival de $86 \%$ pour un réchauffement de $3.3^{\circ} \mathrm{K}$ mais, d'autre part, la proportion des fortes pentes, impropres au déroulement des processus géomorphologiques impliqués dans la formation de sols polygonaux, augmentera fortement aux étages nouvellement disponibles (Theurillat et Guisan 2001). Les sols polygonaux pourraient donc être utiles comme bioindicateurs des changements climatiques. La poursuite de leur étude phytosociologique aidera à mettre en évidence ces changements, et plus généralement, à valoriser notre patrimoine végétal.

Nos remerciements s'adressent spécialement à Jean-Paul Theurillat pour ses précieux conseils sur une version antérieure du présent travail. Merci aux deux experts inconnus pour leurs judicieuses remarques Merci finalement à Jean-Pierre Gyger pour la traduction anglaise. L'article est dédié au Professeur Camille Gervais (1933-2002) en témoignage d'amicale reconnaissance pour tout ce qu'il nous a appris. 


\section{References}

Béguin C. 2006. De l'usage des cartes phytosociologique et géomorphologique pour la conceptualisation des unités de paysage végétal (Sigmeta). Exemple dans la région de Lona (Valais Suisse). Doc. Phytosociol. 20, à l'impression.

Béguin C. et Theurillat J.-P. 1981a. Notes floristiques et phytosociologiques sur la région d'Aletsch. Bull. Murith. 97: 43-70.

Béguin C. et Theurillat J.-P. 1983. Une association végétale des zones humides périglaciaires de l'étage alpin sur silice : le Salici herbaceae-Caricetum lachenalii. Bull. Murith. 99: 33-60.

Beniston M. (ed.)1994. Mountain Environment in Changing Climates. Routledge, London.

Beniston M., Fox D.G., Adhikary S., Andresson R., Guisan A., Holten J.I., Ines J., Maitima J., Price M., Tessier L. (lead authors). 1996. The Impact of Climate Change on Mountain Regions. In: IPCC (eds). Second Assessment Report of the Intergovernmental Panel on Climate Change, Chapter 5. Cambridge University Press. Cambridge.

Braun-Blanquet J. 1964. Pflanzensoziologie. Springer, Wien.

Brink V.C., Ross Macray J., Freyman S. and Pearce D.G. 1967. Needle ice and seedling establishment in southwestern Britisch Columbia. Can. J. Plant Sci. 47:135-139.

Campiche M.P. 1986. Cartographie et étude de phénomènes périglaciaires dans les Hautes Alpes Calcaires. Mémoire de licence, Institut de Géographie, Université de Lausanne, Suisse.

Cannone N., Guglielmin M. and Gerdol R. 2004. Relationships between vegetation patterns and periglacial landforms in northwestwern Svalbard. Polar Biol. 27: 562-571.

Duchaufour P. 1970. Précis de Pédologie. Masson, Paris.

French H. M. 1996. The Periglacial Environnment. 2nd Ed. Longman, England.

Gerber E. 1994. Geomorphologie und Geomorphodynamik der Region Lona-Sasseneire (Wallis, Schweizer Alpen) unter besonderer Berücksichtigung von Lockersedimenten mit Permafrost. Thèse de doctorat N¹060, Institut de Géographie, Université de Fribourg, Suisse.

Gold W.G. and Bliss L.C. 1995. Water limitations and plant community-development in a polar desert. Ecology 76: 1558-1568.

Grabherr G. und Mucina L. (Hrsg.) 1993. Die Pflanzengesellschaften Österreichs. Teil II. Fischer, Jena.

Guinochet M. 1973. Phytosociologie. Masson, Paris.

Guisan A., Theurillat J.-P. and Kienast F. 1998. Predicting the potential distribution of plant species in an alpine environment. J. Veg. Sci. 9: 65-74.

Guisan A. and Theurillat J.-P. 2000. Equilibrium modeling of alpine plant distribution: how far can we go? Phytocoenol. 30: 353-384.

Haeberli W. and Beniston M. 1998. Climate change and its impacts on glaciers and permafrost in the Alps. Ambio 27: 258-265.

Jonasson S. and Sköld S.E. 1983. Influences of frost-heaving on vegetation and nutrient regime of polygon-patterned ground. Vegetatio 53: 97-112.

Körner C. 2003. Alpine Plant Life. Springer, New York.

Landolt E. 1977. Ökologische Zeigerwerte zur Schweizer Flora. Veröff. Geobot. Inst. ETH 64: $1-207$.

Lauber K. und Wagner G. 2001. Flora Helvetica. Haupt, Bern.

Oberdorfer E. 1994. Pflanzensoziologische Exkursionsflora, ed. 7. Ulmer, Stuttgart.

Pissart A. 1973. L'origine des sols polygonaux et striés du Chambeyron (Basses Alpes). Résultats d'expériences de terrain. Bull. Soc. Géogr. Liège. 9: 33-53.

Pissart A. 1987. Géomorphologie périglaciaire. Edition Laboratoire de Géomorphologie et de Géologie du Quaternaire. Liège.

Raemy R. et Huber H. 1990. Erosion de la rive sud du lac de Neuchâtel. Eau, Energie, Air 82: 286-290.

Richard J.L., Bressoud B., Buttler A., Duckert O. et Gallandat J.D. 1993. Carte de la végétation de la région Val de Réchy-Sasseneire (objet CPN 3. 77, Alpes valaisannes, Suisse). Bull. Murith. 111: 9-40.

Smith D.J. 1987. Frost-heave activity in the Mount Rae area, Canadian Rocky Mountains. Arct. Alp. Res. 19: 155-166. 
Tenthorey G. 1993. Paysages géomorphologiques du Haut-Val de Réchy (Valais, Suisse) et hydrologie liée aux glaciers rocheux. Thèse de doctorat $\mathrm{N}^{\circ} 1044$, Institut de Géographie, Université de Fribourg, Suisse.

Theurillat J.-P. 1992. Etude et cartographie du paysage végétal (symphytocoenologie) dans la région d'Aletsch (Valais, Suisse). Beitr. Geobot. Landesaufn. Schweiz 68: 1-384.

Theurillat J.-P. et Matthey. E. 1987. Le Vallon de l'Allondon, promenade botanique. Série documentaire 22 des Conservatoire et Jardin botaniques de la Ville de Genève.

Theurillat J.-P., Aeschimann D., Küpfer P. and Spichiger R. 1995. The higher vegetation units of the Alps. Coll. Phytosociol. 23: 189-239.

Theurillat J.-P., Felber F., Geissler P., Gobat J.-M., Fierz M., Fischlin A., Küpfer P., Schlüssel A., Velutti C. and Zhao G.-F. 1998. Sensitivity of plants and soils in ecosystems of the Alps to climate change. In: Cebon P., Dahinden U., Davies H.C., Imboden D. and Jaeger C.C. (eds.) Views from the Alps: Regional perspectives on Climate Change. MIT Press, Cambridge, MA. 225-308.

Theurillat J.-P. and Guisan A. 2001. Potential impact of climate change on vegetation in the European Alps : a review. Climatic Change 50: 77-109; 53: 529-530.

Wasburn A.L. 1979. Geocryology, a survey of periglacial processes and environnments. 2nd ed. Edward Arnold, London.

Annexe: localisation des relevés

Tableau 1

Relevé 1. Lac de Lona. 607,575/110,375. Commune de Grimentz

Relevé 2. Val de Réchy.605,875/113,750. Commune de Nax

Relevé 3. Val de Réchy. 605,500/112,850. Commune de Nax

Relevé 4. Pointe de la Plaine Morte. 603,475/135,425. Commune de Montana

Relevé 5. Col des Outannes. 604,800/135,225. Commune de Montana

Relevé 6. Grand Galibier. 298,800/4992,325. Maurienne, France

Relevé 7. Sasseneire. 606,200/110,800. Commune de Saint-Martin

Relevé 8. Becs de Bosson. 605,950/112,625. Commune de Saint-Martin

Relevé 9. Becs de Bosson. 605,925/112,625. Commune de Saint-Martin

Tableau 2

Relevé 1. Wyssesee, pt. 2337. 618,650/135,350. Commune de Feschel-Guttet

Relevé 2. Pas de Lovegno. 604,125/113,900. Commune de Chalais

Relevé 3. Le Louché.605,200/113,275. Commune de Nax

Relevé 4. Le Louché. 605,125/113,125. Commune de Nax

Relevé 5. Becs de Bosson. 605,125/113,125. Commune de Nax

Relevé 6. Le Louché. 605,050/113,125. Commune de Nax

Tableau 3

Relevé 1. Becs de Bosson. 605.950/112,625 (relevé 8, tab. I). Commune de Saint-Martin

Relevé 2. Val de Réchy. 605,875/113,750 (relevé 2, tab. I). Commune de Nax

Relevé 3. Lac de Lona. 6o7,575/110,375 (relevé 1, tab. I). Commune de Grimentz

Relevé 4. Pas de Lona. 606,150/112,200. Commune de Saint-Martin

Relevé 5. 606,300/112,500. Commune de Grimentz

Relevé 6. 606,800/112,475. Commune de Grimentz

Relevé 7. Le Louché. 605,075/113,425. Commune de Nax

Relevé 8. Le Louché. 605,125/113,125 (relevé 4, tab. II). Commune de Nax

Relevé 9. Becs de Bosson. 605,125/113,125 (relevé 3, tab. II). Commune de Nax

Relevé 10. Le Louché. 605,200/113,275 (relevé 5, tab. II). Commune de Nax

Relevé 11 Becs de Bosson. 605,025/113,100 (relevé 6, tab. II). Commune de Nax 